\title{
Dieta de juvenis de Trachinotus carolinus (Actinopterygii, Carangidae) em praias arenosas na costa do Rio de Janeiro
}

\author{
Tania Marcia S. Niang, André Luiz M. Pessanha \& Francisco Gerson Araújo
}

Laboratório de Ecologia de Peixes, Departamento de Biologia Animal, Universidade Federal Rural do Rio de Janeiro, km 47 da Antiga Estrada Rio-São Paulo, 23851-970 Seropédica, RJ, Brasil. (gerson@ufrrj.br)

\begin{abstract}
Diet of juvenile Trachinotus carolinus (Actinopterygii, Carangidae) in sandy beaches on coast of Rio de Janeiro, Brazil. We studied the diet of juvenile Trachinotus carolinus (Linnaeus, 1766) in sandy beaches of Sepetiba Bay (Rio de Janeiro, Brazil), between January 2000 and April 2001. We tried to evaluate the trophic plasticity of fish this species along a spatial gradient of wave exposure, seasonality, besides to evaluate ontogenetic changes in the diet. The Index of Relative Importance (IRI) was used to measure the food items, determined by their frequency of occurrence, numbers and weights. The subphylum Crustacea, mainly the order Mysidacea, Decapoda Emerita brasiliensis (Schmitt, 1935) and Cephalochordata, represented by Branchiostoma platae (Fitzinger, 1862) were dominant in the diet. In the most exposed zone (Barra de Guaratiba beach) with predominant sandy substrate, the diet was comprised mainly by Emerita brasiliensis and Cirripedia, this later item common in rocky shores at the beach edge; in the intermediate exposed beach (Muriqui beach), Mysidacea and Branchiostoma platae; in the most protected beach (Itacuruçá beach), Polychaeta, Mysidacea and Branchiostoma platae was predominant. No seasonal change was recorded for the use of Mysidacea, while Branchiostoma platae was the more consumed during the winter, Polychaeta in spring, Cirripedia and Emerita brasiliensis, in summer. Mysidacea was the predominate food in all size classes, while Polychaeta was used predominantly by fish smaller than $20 \mathrm{~mm}$ standard length (SL) and Emerita brasiliensis and Cirripedia, were consumed mainly by larger individuals than $40 \mathrm{~mm}$ in the most exposed beach only. The success in use of surf zones and sand beaches by this fishes species, it can be partly, due the opportunist trophic strategy that uses a wide variety of available resources on environmental.
\end{abstract}

KEYWORDS. Feeding index, pompano, marine fishes, trophic ontogeny, surf zone.

RESUMO. Estudamos a dieta dos juvenis de Trachinotus carolinus (Linnaeus, 1766) em praias da Baía de Sepetiba (Rio de Janeiro, Brasil) entre janeiro de 2000 e abril de 2001. Procuramos avaliar a plasticidade trófica de peixes desta espécie ao longo de um gradiente espacial com diferentes níveis de exposição às ondas, sazonalidade, além de avaliar mudanças ontogenéticas na dieta. Os itens alimentares foram analisados através do índice de importância relativa (IIR), determinado pelos valores das frequências de ocorrência, de número e de peso. Os itens de maior importância foram do subfilo Crustacea, ordens Mysidacea, e o representante da ordem Decapoda Emerita brasiliensis (Schmitt, 1935), além de Cefalochordata, representado por Branchiostoma platae (Fitzinger, 1862). Na zona de maior exposição às ondas (praia de Barra de Guaratiba) e com substrato predominantemente arenoso, a dieta foi constituída principalmente por Emerita brasiliensis e Cirripedia, este último presente nos costões rochosos que limitam a praia; na zona de exposição intermediária (praia de Muriqui), houve um predomínio de Mysidacea e Branchiostoma platae; na zona mais protegida (praia de Itacuruçá), os itens de maior abundância foram Polychaeta, Mysidacea e Branchiostoma platae. Sazonalmente não ocorreu variação no uso de Mysidacea, enquanto Branchiostoma platae foi mais consumido durante o inverno, Polychaeta na primavera e Cirripedia e Emerita brasiliensis, no verão. Mysidacea foi o alimento predominante em todas as classes de tamanho, enquanto Polychaeta foi utilizado predominantemente por peixes menores que $20 \mathrm{~mm}$ de comprimento padrão e Emerita brasiliensis e Cirripedia foram consumidos principalmente por indivíduos maiores que $40 \mathrm{~mm}$, somente na praia de maior exposição. O sucesso no uso de praias desprotegidas e zonas de arrebentação por esta espécie de peixe pode ser em parte devido à estratégia trófica oportunista, que utiliza uma ampla variedade de recursos disponíveis no ambiente.

PALAVRAS-CHAVE. Índice alimentar, pampo, peixes marinhos, ontogenia trófica, zona de arrebentação.

Praias arenosas são caracterizadas como ambientes dinâmicos, especialmente nas zonas de arrebentação. Muitas espécies de peixes utilizam esta área durante suas fases iniciais de vida, tirando proveito da proteção contra seus predadores bem como da alimentação disponível neste ambiente de águas rasas e turbulentas (LASIAK, 1984; Auyvazian \& Hyndes, 1995; Clark et al., 1996). Dentre as espécies adaptadas para exploração das zonas de arrebentação destaca-se a família Carangidae, especialmente os representantes do gênero Trachinotus (Linnaeus, 1766), popularmente conhecidos como pampos. No sudeste do Brasil o gênero Trachinotus é representado por quatro espécies: Trachinotus carolinus (Linnaeus, 1766), T. falcatus (Linnaeus, 1758), T. goodei (Jordan \& Evermann, 1896) e T. marginatus (Curvier, 1832), das quais apenas T. carolinus e T. falcatus foram registradas para a Baía de Sepetiba, Rio de Janeiro e praias arenosas adjacentes, sendo a primeira espécie predominante (ARAúso et al., 1997; Pessanha et al., 2000).

Altas abundâncias de pampos têm sido registradas em diferentes estudos em zonas de arrebentação ao longo da costa brasileira (BARREIROs et al., 2004; VASCONCELLOS et al., 2007; Felix et al., 2007), com indicações que $T$. carolinus apresenta respostas às características físicas e biológicas deste ambiente. A ação contínua das ondas sobre o fundo arenoso embora estressante, disponibiliza grande quantidade de alimento, permitindo a captura de presas neste ambiente por espécies que conseguem adaptar-se a estas condições (CLARK, 1997). Este parece ser o caso da espécie $T$. carolinus que utiliza o ambiente da zona de arrebentação como área de recrutamento e alimentação (LAYMAN, 2000).

As características morfológicas são fatores importantes para a utilização da zona de arrebentação 
pelos peixes (McCoRMICK, 1998), permitindo às espécies que vivem nesta área revolver eficazmente a areia para atacar organismos bênticos. O estudo de ZAHORCSAK et al. (2000) numa praia arenosa brasileira relaciona a morfologia externa de peixes bentívoros com o comportamento forrageiro e fuga de predadores, permitindo que estes peixes explorem eficientemente substratos macios, tais como as costas arenosas. Como características morfológicas de $T$. carolinus são destacadas o focinho caracteristicamente rombudo, boca subterminal e protuberante que facilita a captura das presas no substrato (Menezes \& Figueiredo, 1980); a nadadeira caudal fortemente furcada para facilitar o deslocamento e maior rapidez natatória na zona de arrebentação e as brânquias amplamente espaçadas que permitem a passagem dos grãos de areia e podem reter as presas maiores (BELLINGER \& AvAULT, 1971).

Alguns membros do gênero Trachinotus têm atraído particular atenção por apresentarem um potencial para maricultura (MonTEIRO-NeTO \& CunHA, 1990; BATISTIC $e t$ al., 2005), levando à investigação da sua dieta. Alguns autores, em outros países, têm observado que pampos juvenis se alimentam de poliquetas (BELLINGER \& Avault, 1971) e pequenos crustáceos como Amphipoda, Copepoda e Mysidacea (Astarloa et al., 2000), apresentando variações sazonais e ontogenéticas na dieta (Chervinski \& Zorn, 1977; Armitage \& Alevison, 1980). No Brasil, Helmer et al. (1995) investigaram a dieta de três espécies deste gênero, observando uma grande sobreposição tanto temporal quanto por grupo de tamanho na dieta. O pampo T. falcatus consumiu principalmente Amphipoda, enquanto T. goodei e $T$. carolinus diferiram na composição da dieta entre seus principais crustáceos, Mysidacea e Amphipoda, respectivamente. Monteiro-Neto \& Cunha (1990) estudaram a dieta de T. marginatus na praia do Cassino, Rio Grande (RS), e verificaram que indivíduos menores alimentam-se primariamente de pequenos crustáceos planctônicos e bênticos, enquanto os juvenis, de poliquetas até insetos.

O presente trabalho teve como objetivo descrever a alimentação de juvenis de T. carolinus em praias arenosas da Baía de Sepetiba, Rio de Janeiro. Foi investigada a adequabilidade da área para desenvolvimento/alimentação dessa espécie e testou-se a hipótese de que a variabilidade espacial na dieta depende do grau de exposição às ondas. Além disso, foi avaliada a dieta dos peixes em diferentes classes de comprimento, assim como os efeitos das estações do ano sobre a dieta desses peixes.

\section{MATERIAL E MÉTODOS}

Este estudo foi conduzido em três praias arenosas nas proximidades da Baía de Sepetiba, localizada no

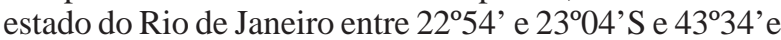
$44^{\circ} 10^{\prime} \mathrm{W}$ (BRASIL, 1984) (Fig. 1). Essas praias foram escolhidas por representarem diferentes graus de exposição aos batimentos de ondas. Como representante do local mais exposto, foi escolhida a praia de Barra de Guaratiba, situada externamente à baía; Muriqui foi o local representativo de uma situação de exposição intermediária, situado na baía, porém, próximo da zona de maior influência do mar; Itacuruçá foi escolhida como a praia arenosa mais protegida devido a proximidades desta com a ilha de Itacuruçá, que constitui uma proteção natural, diminuindo a intensidade das ondas e fazendo com que as águas sejam mais tranquilas naquela região (Fig. 1). De acordo com McLachlan (1980), a praia de Barra de Guaratiba é considerada de exposição "alta" e as praias de Muriqui e Itacuruçá como "baixa". Os fatores ambientais, como salinidade e temperatura, apresentam um gradiente que caracteriza as três praias: na mais exposta são registradas maior salinidade (34) e menor temperatura $\left(22,8^{\circ} \mathrm{C}\right)$ e nas menos expostas, menor salinidade $\left(32,3\right.$ e $\left.29,1^{\circ} \mathrm{C}\right)$ e maior temperatura $(24,5$ e $26,2^{\circ} \mathrm{C}$ ), respectivamente (AraúJo et al., 1997; Pessanha et al., 2000).

Os peixes foram capturados através de arrastos de praia realizados mensalmente de janeiro a dezembro de 2000 e em abril de 2001. Os arrastos foram conduzidos durante o dia utilizando-se uma rede do tipo picaré, medindo $10 \mathrm{~m}$ de comprimento, $2 \mathrm{~m}$ de altura e malha de $7 \mathrm{~mm}$ de distância entre-nós. Em cada praia foram feitas três repetições de cada arrasto.

Os peixes coletados foram fixados em formalina a $10 \%$ no campo, com posterior conservação em álcool $70 \%$ em laboratório, após 48 horas da fixação. A identificação foi feita de acordo com Menezes \& Figueiredo (1980). De cada exemplar foi medido o comprimento padrão (do extremo do focinho até o inicio da nadadeira caudal) em milímetros, além do peso total em gramas.

O conteúdo estomacal de 300 espécimes de $T$. carolinus foi analisado em microscópico estereoscópio, sendo cada item separado, contado e pesado em uma balança de precisão de $0,01 \mathrm{~g}$. Todos os itens foram identificados até o nível taxonômico mais inferior possível. Foi aplicado o Índice de Importância Relativa (IIR), que engloba os métodos de frequência de ocorrência (\% FO), frequência numérica ( $\% \mathrm{FN})$ e frequência de peso $(\% \mathrm{FP})$ dos itens alimentares, onde o IIR $=(\% \mathrm{FN}+\% \mathrm{FP}) \times \% \mathrm{FO}$ (PINKAS, 1971).

Os itens alimentares encontrados nos estômagos dos peixes foram analisados em relação aos locais de

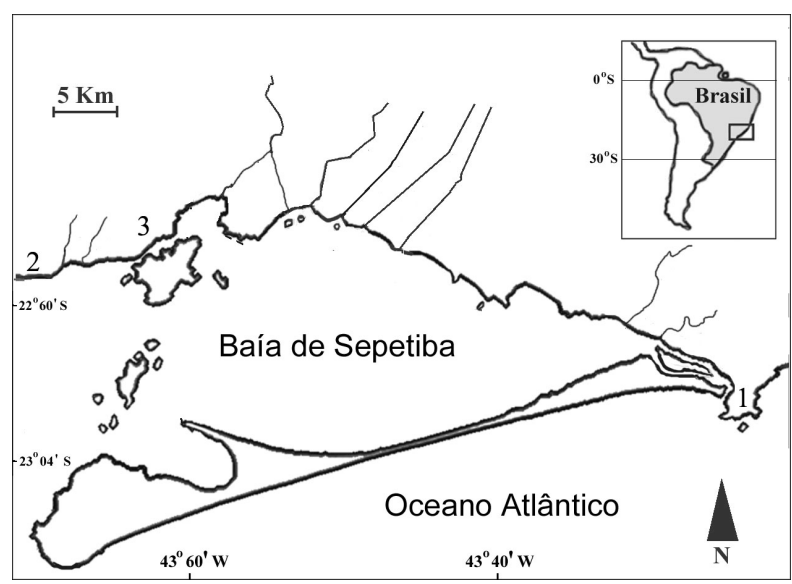

Figura 1. Área de estudo, Baía de Sepetiba, RJ, com indicação dos pontos de coleta: 1, Barra de Guaratiba; 2, Muriqui; 3, Itacuruçá. 
coleta (praias com diferentes níveis de exposição), bem como em relação às variações sazonais e de tamanho dos indivíduos. Para a análise sazonal da dieta, os dados foram agrupados em quatro estações: primavera (setembronovembro); verão (dezembro-fevereiro); outono (marçomaio) e inverno (junho-agosto). Para o estudo do tamanho, os peixes foram divididos em quatro classes de comprimento padrão: Classe I = menor que $20 \mathrm{~mm}$; Classe II $=$ de 21 a $40 \mathrm{~mm}$; Classe III= de 41 a $60 \mathrm{~mm}$; Classe IV= maior que $60 \mathrm{~mm}$.

\section{RESULTADOS}

Dieta geral. Foram analisados 300 estômagos de $T$. carolinus sendo que destes, $242(80,7 \%)$ tiveram seus conteúdos efetivamente examinados e $58(19,3 \%)$ encontravam-se vazios (Tab. I). Os estômagos que continham apenas material digerido (34) ou o item Cirripedia (17) somente foram avaliados quanto a frequência de ocorrência e peso, não sendo incluídos no cálculo do Índice de Importância Relativa (Tab. I).

Seis categorias de itens alimentares foram identificadas para T. carolinus: Polychaeta, Crustacea, Insecta, Cephalocordata, Chordata e outros materiais. Crustacea foi a categoria de maior importância na dieta, com 8 itens, com destaque para Mysidacea e Emerita brasiliensis (Schmitt, 1935) (Tab. I).

A dieta de T. carolinus foi baseada em itens encontrados em toda a coluna d'água, além de itens alóctones como Hymenoptera e penas de aves. Os maiores valores do IIR foram registrados para os itens ligados ao substrato, como Mysidacea (IIR=5.117), $E$. brasiliensis (IIR=1.393) e Branchiostoma platae (Fitzinger, 1862) (IIR=138,9) (Tab. I).

Variação espacial na dieta. Houve variação na dieta entre as praias estudadas. Na praia mais desprotegida (Barra de Guaratiba), com base na análise dos 135 estômagos, a dieta consistiu de quatro itens principais: E. brasiliensis, ovos de peixe, Polychaeta e Hymenoptera (Fig. 2). O item E. brasiliensis apresentou a maior contribuição no IIR, com o valor de 7.820; outros itens alimentares tiveram IIR relativamente baixo como ovos de peixe (IIR=259), Hymenoptera (IIR=21,6) e Polychaeta (IIR=19,9). Apesar de não ter sido possível avaliar o IIR para Cirripedia, o mesmo apresentou elevadas contribuições na frequência de ocorrência $(45,6 \%)$, e frequência de peso $(42,3 \%)$, enquanto material digerido apresentou uma frequência de ocorrência de $33,6 \%$ e $16,7 \%$ de frequência de peso. Vale ressaltar que, apesar do alto valor numérico para o item alimentar ovos de peixes, este não foi frequente nos estômagos analisados.

Os itens alimentares de maior importância relativa na praia de Muriqui foram apenas dois: Mysidacea e B. platae (Fig. 2). O item Mysidacea teve a contribuição no IIR com o valor de 13.598 , enquanto $B$. platae apresentou IIR $=359,4$. Material digerido ocorreu em $37,39 \%$ dos estômagos com alimento contribuindo com $7,5 \%$ do peso.
A dieta de $T$. carolinus na praia de Itacuruçá apresentou maior diversidade e equitabilidade dos itens (Fig. 2), com cinco principais itens sendo registrados: Mysidacea, B. platae, Polychaeta, Hymenoptera e Isopoda. Mysidacea foi o item dominante apresentando IIR=1.189, seguido por Polychaeta com IIR $=1.449, B$. platae com IIR=1.024, Hymenoptera com IIR $=410,8$ e Isopoda com IIR=32. Material digerido teve grande participação nas frequiências, ocorrendo em $63,3 \%$ dos estômagos com alimento e apresentando 50,6\% do peso.

Variação sazonal na dieta. A maior variedade dos itens alimentares ocorreu na primavera, ainda que observado a dominância de Mysidacea (Fig. 3).

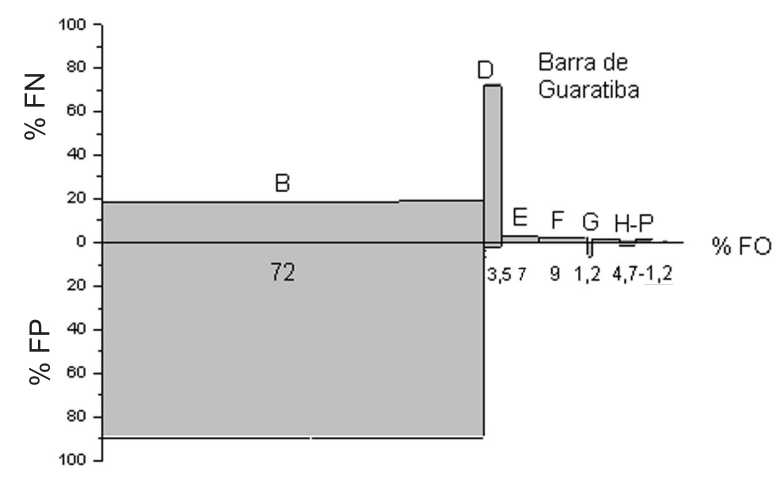

A
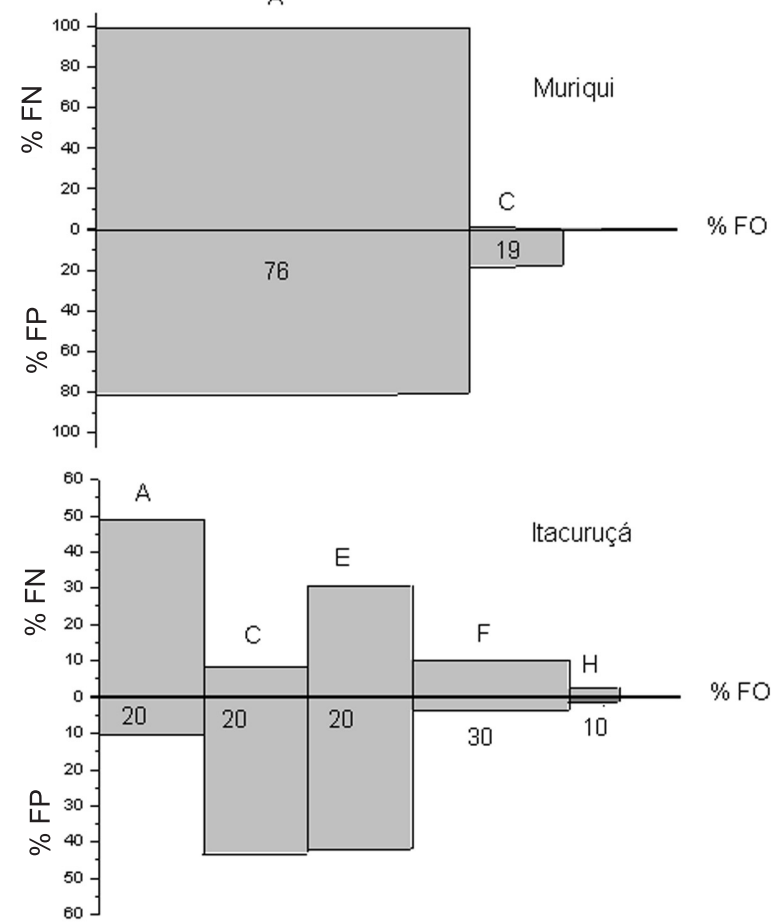

Figura 2. Composição percentual dos itens alimentares dos juvenis de Trachinotus carolinus (Linnaeus, 1766) coletados nos arrastos de praia em Barra de Guaratiba, Muriqui e Itacuruçá entre janeiro/ 2000 e abril/2001, representadas por número (\% FN), peso (\% FP) e frequência de ocorrência (\% FO). Índice de Importância Relativa (IIR) é representado pela área dos retângulos (A, Mysidacea; B, Emerita brasiliensis (Schmitt, 1935); C, Branchiostoma platae (Fitzinger, 1862); D, ovos; E, Polychaeta; F, Hymenoptera; G, pena; H, Isopoda; I, Amphipoda; J, Caprellidae; K, peixe; L, material vegetal; M, Copepoda; N, alga; O, larva de crustáceo; P, Peracarida). 
Tabela I. Itens alimentares encontrados no conteúdo estomacal de Trachinotus carolinus (Linnaeus, 1766) coletados nos arrastos de praia em Barra de Guaratiba, Muriqui e Itacuruçá, RJ, entre janeiro/2000 e abril/2001 (FO, frequência de ocorrência; FN, frequência numérica; FP, frequência de peso; IIR, Índice de Importância Relativa).

\begin{tabular}{|c|c|c|c|c|}
\hline Categorias e Itens alimentares & FO $(\%)$ & FN (\%) & FP $(\%)$ & IIR \\
\hline Polychaeta & 5,80 & 0,70 & 1,30 & 11,46 \\
\hline \multicolumn{5}{|l|}{ Crustacea } \\
\hline Mysidacea & 40,31 & 84,27 & 42,70 & $5.117,00$ \\
\hline \multicolumn{5}{|l|}{ Decapoda } \\
\hline Emerita brasiliensis (Schmitt, 1935) & 32,50 & 2,70 & 40,24 & $1.393,00$ \\
\hline Isopoda & 3,70 & 0,20 & 0,18 & 1,40 \\
\hline Amphipoda não Caprellidae & 2,09 & $<0,10$ & 0,45 & 1,00 \\
\hline Amphipoda Caprellidae & 1,60 & 0,17 & 0,16 & 0,52 \\
\hline Copepoda & 0,52 & $<0,10$ & $<0,10$ & $<0,10$ \\
\hline Tanaidacea & 0,52 & $<0,10$ & $<0,10$ & $<0,10$ \\
\hline Cirripedia & 19,70 & & 24,90 & \\
\hline \multicolumn{5}{|l|}{ Insecta } \\
\hline Hymenoptera & 8,40 & 0,46 & 0,43 & 7,50 \\
\hline \multicolumn{5}{|l|}{ Cephalochordata } \\
\hline Branchiostoma platae (Fitzinger, 1862) & 12,04 & 0,85 & 10,70 & 138,90 \\
\hline \multicolumn{5}{|l|}{ Chordata } \\
\hline Ovos de peixes & 1,60 & 10,40 & 0,68 & 17,38 \\
\hline Peixes & 1,60 & $<0,10$ & 0,10 & 0,32 \\
\hline Larvas de peixe & 0,52 & $<0,10$ & $<0,10$ & $<0,10$ \\
\hline \multicolumn{5}{|l|}{ Outros materiais } \\
\hline Pena de aves & 1,00 & $<0,10$ & 2,82 & 3,00 \\
\hline Material vegetal & 1,60 & $<0,10$ & $<0,10$ & 0,20 \\
\hline Algas & 0,52 & $<0,10$ & $<0,10$ & $<0,10$ \\
\hline Material digerido & 37,60 & & 14,50 & \\
\hline
\end{tabular}
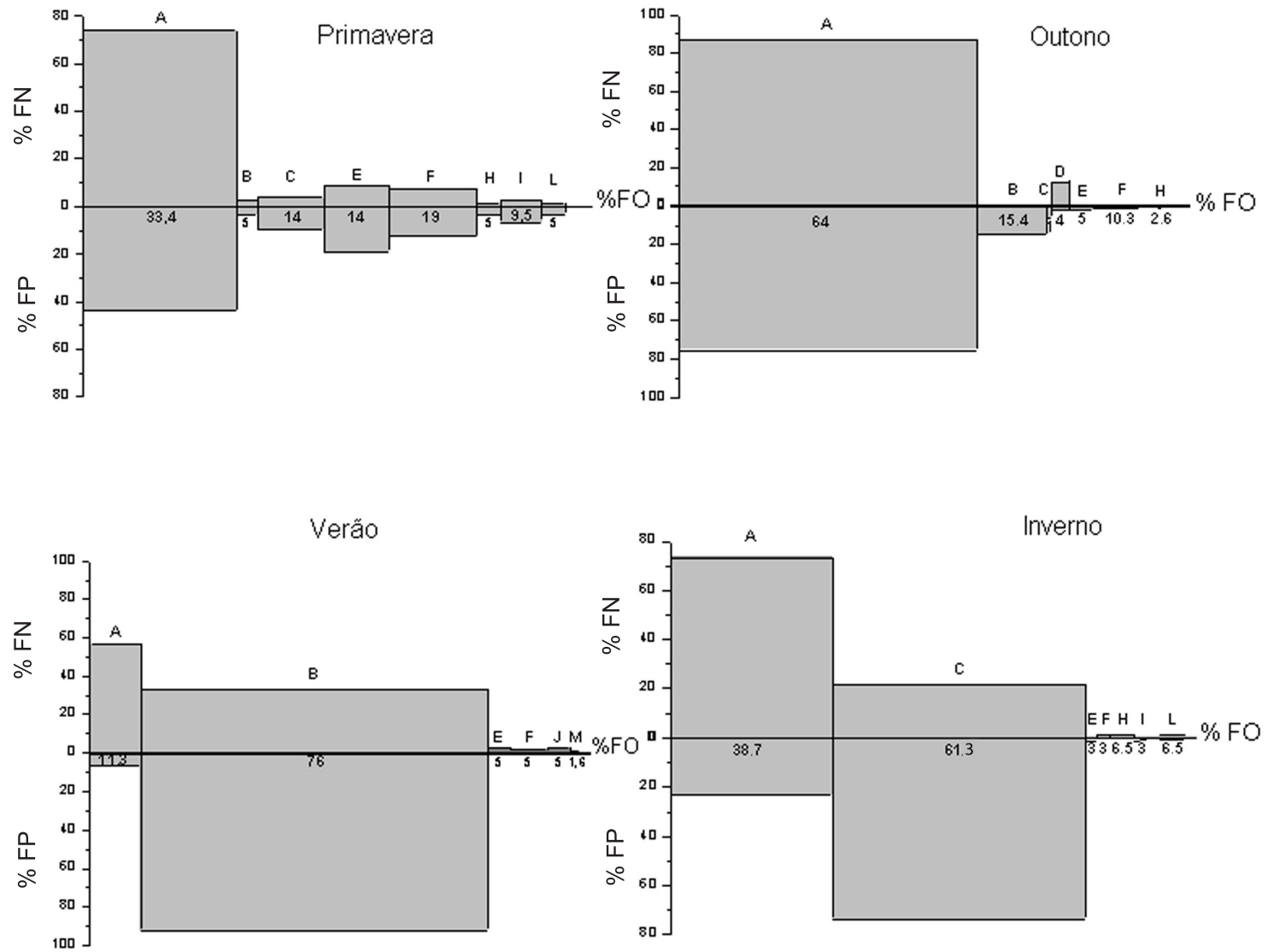

Figura 3. Composição percentual dos itens alimentares dos juvenis de Trachinotus carolinus (Linnaeus, 1766) durante as estações do ano representadas por número $(\% \mathrm{FN})$, peso $(\% \mathrm{FP})$ e frequência de ocorrência (\% FO). Índice de Importância Relativa (IIR) é representado pela área dos retângulos (A Mysidacea; B, Emerita brasiliensis (Schmitt, 1935); C, Branchiostoma platae (Fitzinger, 1862); D, ovos; E, Polychaeta; F, Hymenoptera; G, pena; H, Isopoda; I, Amphipoda; J, Caprellidae; K, peixe; L, material vegetal; M, Copepoda; N, alga; O, larva de crustáceo; P, Peracarida). 
Os principais itens alimentares presentes nesta estação do ano foram: Mysidacea com contribuição de IIR=3.928,9; Hymenoptera contribuindo com IIR=372,6; Polychaeta com IIR $=385,5 ;$ B platae de IIR $=184,35$ e $E$. brasiliensis $\mathrm{IIR}=26$. $\mathrm{O}$ item material digerido apresentou ocorrência de 41,7 \% nos estômagos que continham alimento e uma participação de $71,9 \%$ no peso total.

No verão $(\mathrm{n}=99)$ as categorias alimentares mais importantes foram E. brasiliensis e Mysidacea: o primeiro item contribuiu com IIR=9.436 (Fig. 3) enquanto que o segundo com IIR=713,3. O item material digerido apresentou ocorrência de $28 \%$ nos estômagos que continham alimento e uma participação de $4,6 \%$ no peso total, enquanto que o item Cirripedia teve uma grande participação com 60,6 \% de ocorrência em estômagos com alimento e $47,2 \%$ de presença no peso destes estômagos.

No outono as categorias alimentares mais importantes foram: Mysidacea contribuindo com IIR=10.385; E. brasiliensis com IIR=228,5 e ovos de peixe contribuindo com IIR=50,5. O item material digerido apresentou ocorrência de 43,7\% nos estômagos que continham alimento e uma participação de $23 \%$ no peso total (Fig. 3). O item Cirripedia não apareceu nesta estação.

No inverno (n=56) (Fig. 3) a dieta de T. carolinus consistiu basicamente em duas categorias principais: $B$. platae, contribuindo com IIR=5.862,7 e Mysidacea, contribuindo com IIR=3.747,9. $\mathrm{O}$ item material digerido apresentou ocorrência de $40 \%$ nos estômagos que continham alimento e uma participação de $15 \%$ no peso total. O item Cirripedia não apareceu nos conteúdos estomacais nesta estação do ano.

Variação ontogenética na dieta. Os espécimes de $T$. carolinus analisados apresentaram comprimento padrão variando entre 12 a $83 \mathrm{~mm}$. Com base no hábito alimentar dessa espécie foi verificada uma mudança na dieta entre as diferentes classes de tamanho. O item Mysidacea é utilizado em todas as classes enquanto $E$. brasiliensis é utilizada nas classes de maior tamanho.

Nos indivíduos da Classe I $(<20 \mathrm{~mm})(\mathrm{n}=14)$ a dieta consistiu principalmente em três categorias: Mysidacea que contribuiu com IIR=6.389; Polychaeta com IIR=1.088 e B. platae com IIR=717,6. Material digerido ocorreu em $62,5 \%$ dos estômagos com alimento e 55,6 \% do peso total (Fig. 4).

$\mathrm{Na}$ Classe II $(20-40 \mathrm{~mm})(\mathrm{n}=115)$ a dieta apresentou um maior número de itens alimentares, onde 6 itens dominaram: Mysidacea que contribuiu com IIR=30,28 seguido por Hymenoptera com IIR=248,5; Polychaeta com IIR $=172,3$; ovos de peixe com $\mathrm{IIR}=125,5 ;$ B. platae $\mathrm{com}$ IIR=46 e $E$. brasiliensis $\mathrm{com}$ contribuição de IIR=11,67. Material digerido ocorreu em 43,2 \% dos estômagos com alimento e $39,4 \%$ do peso total (Fig. 4).

$\mathrm{Na}$ Classe III (41- $60 \mathrm{~mm})(\mathrm{n}=130)$ a dieta consistiu basicamente de Mysidacea com IIR=6.469, seguido por E. brasiliensis com IIR=1445 e B. platae com IIR=218 (Fig. 4). Material digerido ocorreu em 29,4\% dos estômagos com alimento e $8,8 \%$ do peso, enquanto o item Cirripedia teve uma participação de $29 \%$ dos estômagos com alimento e $30 \%$ de peso total (Fig. 4).

Nos indivíduos maiores da Classe IV (> $60 \mathrm{~mm}$ ) $(\mathrm{n}=41)$ a dieta consistiu principalmente em quatro categorias (Fig. 4): E. brasiliensis com o IIR=35.574, seguido de Mysidacea com IIR=2.464,8; B. platae $\mathrm{com}$ IIR $=62,6$ e ovos de peixe representado por IIR $=41,8$. Material digerido ocorreu em 46,3\% dos estômagos com alimento e 19,3\% do peso, enquanto o item Cirripedia teve uma participação de 31,2 dos estômagos com alimento e $18,7 \%$ de peso total (Fig. 4).
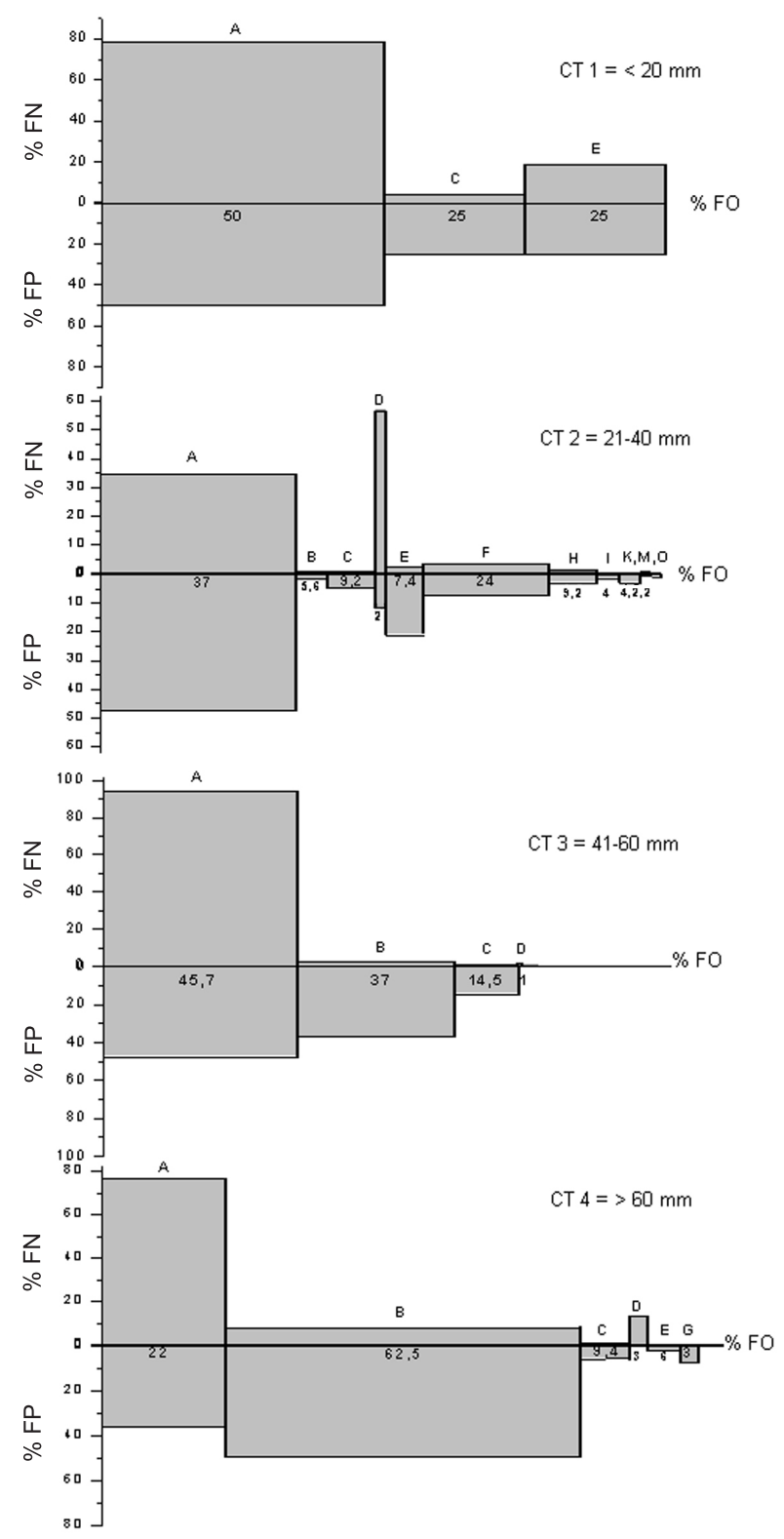

Figura 4. Composição percentual dos itens alimentares dos juvenis de Trachinotus carolinus (Linnaeus, 1766) para as diferentes classes de tamanho, representados por número $(\% \mathrm{FN})$, peso ( $\% \mathrm{FP})$ e frequência de ocorrência (\% FO). Índice de Importância Relativa (IIR) é representado pela área dos retângulos (A, Mysidacea; B, Emerita brasiliensis (Schmitt, 1935); C, Branchiostoma platae (Fitzinger, 1862); D, ovos; E, Polychaeta; F, Hymenoptera; G, pena; H, Isopoda; I, Amphipoda; J, Caprellidae; K, peixe; L, material vegetal; M, Copepoda; N, alga; O, larva de crustáceo; P, Peracarida). 


\section{DISCUSSÃO}

A exposição às ondas pode afetar a alimentação dos peixes devido à modificação da estrutura do sedimento e/ou à influência sobre as fontes de alimento (Bennett, 1989; Clark, 1997), como por exemplo, a zonação dos invertebrados bentônicos. A utilização de organismos de fundo na alimentação de juvenis de pampos tem sido ressaltada em outros sistemas costeiros brasileiros: em praias arenosas de Camburi, Vitória (ES) a dieta de três espécies de Trachinotus foi baseada em moluscos, anfípodos e poliquetas (HELMER et al., 1995); na praia do Cassino (RS), uma zona de arrebentação de elevada energia, a dieta de Trachinotus marginatus foi composta primariamente de pequenos crustáceos Copepoda, Mysidacea e E. brasiliensis (MonteIRo-Neto \& CunHA, 1990). No presente estudo, observamos também o item Mysidacea e E. brasiliensis na dieta de $T$. carolinus, coincidindo com os resultados de MonTEIRONeto \& Cunha (1990), porém diferindo dos de Helmer et al. (1995). Tais diferenças podem ser atribuídas à grande plasticidade trófica deste gênero. Neste trabalho, o nível de exposição às ondas, que variou entre as praias examinadas, foi determinante para obtenção diferenciada de recursos alimentares pelos juvenis de pampos, com $E$. brasiliensis sendo dominante na dieta no local mais exposto e Mysidacea nos locais menos expostos.

A praia de Barra de Guaratiba, localizada numa zona desprotegida, com uma ação forte das ondas e substrato predominantemente arenoso, apresentou além do predomínio de E. brasiliensis, grande participação de Cirripedia presente nos costões rochosos que limitam esta praia. TEIXEIRA et al. (1992) descrevem que em zonas de arrebentação de praias, ondas de média e alta energia contribuem sucessivamente para revolver o fundo e desenterrar muitos organismos, tornando-os acessíveis a predadores que não apresentam grandes habilidades para explorar os organismos da infauna, e tal hipótese explica a exploração de E. brasiliensis por T. carolinus.

Nas praias de Muriqui e Itacuruçá, localizadas em zonas de menor exposição às ondas, houve um predomínio de Mysidacea e de B. platae, sendo que nesta última praia foi verificada a maior variedade de itens alimentares possivelmente devido ao menor nível de exposição. Estes resultados concordam com CARR \& Adams (1973), que também avaliaram a dieta de juvenis $T$. carolinus capturados em estuários, zonas relativamente mais protegidas, e constataram o predomínio de Mysidacea. Locais de menor exposição às ondas podem favorecer a maior diversidade biológica em geral, uma vez que somente poucas espécies são adaptadas para suportar as maiores pressões ambientais causadas pela elevada energia do ambiente, como é o caso de zonas de arrebentação. ROMER (1990) e CLARK (1997) sugerem que a alta exposição às ondas é geralmente acompanhada por um decréscimo na abundância de peixes e invertebrados e por um aumento na dominância de um pequeno número de espécies.

Um outro aspecto que influenciou o comportamento alimentar e a dieta dessa espécie é a presença de competidores ou predadores nas praias estudadas. Das espécies que partilham o mesmo habitat que $T$. carolinus podemos ressaltar Menticirrhus littoralis (Holbrook, 1847) nas praias batidas e Eucinostomus argenteus Baird \& Girard, 1855 e Diapterus rhombeus (Cuvier, 1829) na área protegida (PESSANHA et al., 2000). No entanto, somente dispomos de dados sobre os hábitos alimentares das espécies que partilham a area protegida, com tais espécies consumindo itens alimentares como poliquetas e copepodas, que são bem distintos dos itens principais do pampo (SANTOS \& ARAúJO, 1997). Portanto, não teríamos, entre elas, uma competição por recursos nesta área.

Mysidacea, considerado um abundante recurso nas zonas de arrebentação (LASIAK \& McLACHLAN, 1987; WAKABARA et al., 1993), foi o item predominante na dieta de $T$. carolinus durante todo o ano. Devido à constância ao longo do tempo dessa presa principal, um padrão sazonal na dieta de $T$. carolinus não foi bem claro. As variações observadas foram decorrentes da contribuição dos diferentes itens entre as estações do ano, com dominância do item $E$. brasiliensis no verão e $B$. platae no inverno. Essas variações podem estar relacionadas com a biologia da presa, pois no caso de E. brasiliensis as maiores abundâncias são registradas na primavera/ verão, coincidindo com o pico da reprodução da espécie (Petracco \& Veloso, 2003). A variação sazonal na dieta dessa espécie também é evidenciada por outros trabalhos, como o de HELMER et al. (1995) que encontraram moluscos como principal item na primavera e Amphypoda no verão. Tais resultados não são coincidentes com os do presente trabalho, sugerindo diferenças na disponibilidade das presas ao longo do ciclo anual entre as duas áreas e, por consequência, diferenças na alimentação.

A análise alimentar para as diferentes classes de tamanho de $T$. carolinus evidenciou mudanças ontogenéticas na utilização dos itens alimentares. Organismos como os microcrustáceos da ordem Mysidacea constituem o principal item alimentar nas diferentes classes de tamanho; somente os peixes que ocorrem em zonas expostas das Classes III e IV (> $40 \mathrm{~mm}$ ) passam a utilizar E. brasiliensis, uma presa de tamanho relativamente maior. Algumas espécies de peixes modificam suas estratégias com relação ao tamanho da presa ingerida, onde os adultos investem em presas maiores, em menor número e mais pesadas (Ross, 1978; Teixeira \& Haimovici, 1989) corroborando a hipótese de que indivíduos menores alimentam-se de pequenas presas, enquanto indivíduos maiores utilizam presas maiores (Austin \& Austin, 1971). Crustáceos são a principal fonte de alimento para muitas espécies de peixes, principalmente nas fases iniciais de seus ciclos de vida (Burchmore et al., 1984; WaKABARA et al., 1993). Assim, T. carolinus utiliza os recursos alimentares mais disponíveis no seu habitat, como no caso os Mysidacea, abundantes nas zonas de arrebentação (LASIAK \& McLachlan, 1987). Slobodokin (1961) chama atenção para o fato de que um predador prudente é aquele que atua sobre a presa mais comum em seu habitat. Em geral, o grupo de tamanho entre 20 e $40 \mathrm{~mm}$ é o de maior dominância nas praias arenosas e o que apresentou maior variedade de itens alimentares em relação às classes inferiores ou superiores, indicando o grande oportunismo dessa espécie nessa fase, a qual utiliza essas zonas de arrebentação em sua fase inicial de vida. 
Juvenis de $T$. carolinus utilizaram os itens alimentares disponíveis nas praias arenosas e zonas de arrebentação, com variações observadas em função do batimento dos locais estudados, períodos do ano e desenvolvimento ontogenético. Tais variações indicam a grande plasticidade trófica e oportunismo dessa espécie, com o item Mysidacea tendo sido dominante na maioria das observações. Outros itens de destaque foram E. brasiliensis, somente na praia de mais exposta (Barra de Guaratiba) e B. platae nos locais de menor intensidade das ondas (Itacuruçá e Muriqui).

Agradecimentos. Aos estudantes de graduação e pósgraduação do Laboratório de Ecologia de Peixes da UFRRJ que colaboraram nas coletas e em diversas fases deste trabalho, especialmente à Márcia Cristina C. de Azevedo, Ana Paula Guedes, Rosana Milagre, Iracema D. Gomes, Pablo Mendonça, Rafaela N. Vicentini, Francisco José C. Guimarães e Marcus R. da Costa. Ao $\mathrm{CNPq}$, pela Bolsa de Iniciação Científica ao primeiro autor (Programa PIBIC/UFRRJ), e pelo apoio financeiro para o projeto Bioecologia de Peixes da Baía de Sepetiba (Processos 522317/ 96-0 e 463317/00-9)

\section{REFERÊNCIAS BIBLIOGRÁFICAS}

Araujo, F. G.; Cruz-Filho, A. G.; Azevedo, M. C. C.; Santos, A. C. A. \& Fernades, L. A. M. 1997. Estrutura da comunidade de peixes jovens da margem continental da Baía de Sepetiba, RJ. Acta Biológica Leolpoldensia 19(1):61-83.

Armitage, T. M. \& Alevison, W. S. 1980. The diet of the Florida Pompano (Trachinotus carolinus) along the east coast of central Florida. Florida Science 43:14-19.

Astarloa, J. M. D.; Figueroa, D. E.; Cousseau, M. B \& Barrágan, M. D. 2000. Occurrence of Trachinotus carolinus (Carangidae) in Laguna Costera Mar Chiquita, whit comments on other occasionally recorded fishes in argentinean waters. Bulletin of Marine Science 66:399-403.

Austin, H. \& Austin, S. 1971. The feeding habits of some juvenile marine fishes from the mangroves in western Puerto Rico. Caribbean Journal of Sciences 11:171-178.

Auyvazian, S. G. \& Hyndes, G. A. 1995. Surf-zone fish assemblages in south - western Australia: do adjacent nearshore habitats and warm Leeuwin Current influence the characteristics of the fish fauna? Marine Biology 122:527-536.

Barreiros, J. P.; Figna, V.; Hostim-Silva, M. \& Santos, R. S. 2004 Seasonal changes in sandy beach fish assemblage at Canto Grande, Santa Catarina, South Brazil. Journal of Coastal Research 20:862-870.

Batistic, M.; Tutman, P.; Bojanic, D.; Skaramuca, B.; Kozul, V.; Glavic, N. \& Batulovic, V. 2005. Diet and feeding activity of juvenile pompano (Trachinotus ovatus) (Teleostei: Carangidae) from the southern Adriatic, Croatia. Journal of the Marine Biological Association of the United Kingdon 85:1533,1534.

Bellinger, J. W. \& Avault, J. W. 1971. Food habits of juvenile pompano. Trachinotus carolinus, in Lousiana. Transactions of the American Fisheries Society 100(3):486-494.

Bennett, B. A. 1989. The fish community of a moderately exposed beach on the southwestern cape coast of South Africa and an assessment of this habitat a nursery for juvenile fish. Estuarine Coastal and Shelf Science 28:293-305.

BRASIL (Ministério da Marinha). 1984. Baía de Sepetiba. Carta náutica 1621. Rio de Janeiro, Diretoria de Hidrografia e Navegação.

Burchmore, J. J.; Pollard, D. A. \& Bell, J. D. 1984. Community structure and trophic relationship of the fish fauna of in estuarine Posidonia australis seagrass habitat in Port Haching, New South Wales. Aquatic Botany 18:71-87.

CARr, W. E. \& Adams, C. A. 1973. Food habits of juvenile marine fishes occupying seagrass beds in the estuarine zone near Crystal River, Florida. Transactions of the American Fisheries Society 102:511-540.
Chervinski, J. \& Zorn, M. 1977. Note on occurrence and the food of juvenile kachlan (Trachinotus ovatus, Linaeus, Pisces, Carangidae) from the Mediterranean. Aquaculture 10(2): 179-185.

Clark, B. M. 1997. Variation in Surf-zone Community Structure Across a Wave - exposure gradient. Estuarine Coastal and Shelf Science 44:659-674.

Clark, B. M.; Bennet, S. J. \& Lamberth, S. J. 1996. Factors affecting spatial variability in seine net catches of fish in the surf zone of False Bay, South Africa. Marine Ecology Progress Series 131:35-47.

Felix, F. C.; Spach, H. L.; Moro, P. S.; Junior, R. S.; Santos, C.; Hackradt, C. W. \& Hostim-Silva, M. 2007. Utilization patterns of surf zone inhabiting fish from beaches in Southern Brazil Pan-American Journal of Aquatic Science 1:27-39.

Helmer, J. L.; Teixeira, R. L \& Monteiro-Neto, C. 1995. Food habits of young Trachinotus (Pisces, Carangidae) in the inner surf-zone of a sandy beach in southeast Brazil. Atlântica 17:95-107.

LASIAK, T. A. 1984. Structural aspects of the surf zone fish assemblage at King's Beach, Algoa Bay, South Africa: Long term fluctuations. Estuarine Coastal and Shelf Science 18(4):459-483.

LASIAK, T. A. \& MCLACHLAN, A. 1987. Opportunistic utilization of mysid shoals by surf-zone teleosts. Marine Ecology Progressive 37:1-7.

Layman, C. A. 2000. Fish assemblage structure of the shallow ocean surf-zone on the eastern shore of Virginia Barrier islands. Estuarine Coastal and Shelf Science 51:201213.

McLachlan, A. 1980. The definition of sandy beaches in relation to exposure: a simple system. South African Journal of Science 76:137-138

MCORMICK, M. I. 1998. Ontogeny of diet shifts by microcarnivorous fish, Cheilodactylus spectabilis: relationship between feeding mechanics, microhabitat selection and growth. Marine Biology 132:9-20.

Menezes, N. A. \& Figueiredo, J. L. 1980. Manual de peixes marinhos do sudeste do Brasil. São Paulo, Editora Museu de Zoologia da USP. 63p.

Monteiro-Neto, C. M. \& Cunha, L. P. R. 1990. Seasonal and antogenetic variation in food habitats of juvenile Trachinotus marginatus Cuvier, 1832 (Teleostei, Carangidae) in the surf zone of cassino beach, RS, Brazil. Atlântica 12(1):45-54.

Pessanha, A. L. M.; Araújo, F. G.; Azevedo, M. C. C. \& Gomes, I. D. 2000. Variações temporais e espaciais da comunidade de peixes jovens da Baía de Sepetiba, Rio de Janeiro. Revista Brasileira de Zoologia 17(1):251-261.

Petracco, M. \& Velloso, C. 2003. Secondary production of the intertidal macrofauna of Prainha Beach, Brazil. Journal of Coastal Research 35:385-391.

Pinkas, L. 1971. Food habits study. In: Pinkas L.; Oliphant, M. S. \& Iverson, I. L. K. eds. Foods habitats of albacore bluefin tuna, and bonito in California waters. Monterrey, California Fish Bulletin. v.152, 105p.

Romer, G. S. 1990. Surf zone fish community and species response to wave energy gradient. Journal of Fishes Biology 36: $279-287$

Ross, S. T. 1978. Trophic ontogeny of the leopard searobin, Prinotus scitulus (Pisces: Triglidae). Fisheries Bulletin 76:225-234

Santos, A. C. C. \& Araújo, F. G. 1997. Hábitos alimentares de três espécies de Gerreidae (Osteichthyes, Perciformes) na Baía de Sepetiba. Arquivos de Biologia e Tecnologia 40:359-368. SLobodkin, L. B. 1961. Growth and regulation of animal populations. New York, Holt, Renehart and Winston. 184p.

Teixeira, R. L.; Falcão, G. A. F. \& Melo, S. C. 1992. Ocorrência e alimentação de juvenis de Sciaenidae (Pisces: Perciformes) nas zonas de arrebentação de praias de Maceió, Brasil. Atlântica 4:29-42

Teixeira, R. L. \& Haimovici, M. 1989. Distribuição, reprodução e hábitos alimentares de Prionotus punctatus e P. nudigula (Pisces: Triglidae) no litoral do Rio Grande do Sul, Brasil. Atlântica 11:13-45. 
Vasconcellos, R. M.; Santos, J. N. S.; Silva, M. A. \& Araújo, F. G. 2007. Efeito do grau de exposição às ondas sobre a comunidade de peixes juvenis em praias arenosas do município do Rio de Janeiro, Brasil. Biota Neotropica 7:171-178.

Wakabara, Y.; Tararam, A. S. \& Flynn, M. N. 1993. Importance of the macrofauna for the feding of young fish species from infralittoral of Arrozal-Cananeia lagoon- estuarine region $\left(25^{\circ} 02^{\prime} \mathrm{S}-47^{\circ} 56^{\prime} \mathrm{W}\right)$ - Brazil. Boletim do Instituto Oceanografico 41:39-59.

ZahorcsaK, P.; Silvano, R. A. M. \& SAZIMA, I. 2000. Feeding biology of a guild of benthivorous fishes in a sandy shore on south-eastern brazilian coast. Revista Brasileira de Biologia 60:511-518. 